\title{
MODELAMIENTO DE LA REMOCIÓN DE MATERIA ORGÁNICA EN UN REACTOR ANAEROBIO DE FLUJO ASCENDENTE
}

\author{
MODELING THE REMOVAL OF ORGANIC MATTER IN AN ANAEROBIC REACTOR UPFLOW
}

\author{
Ingaruca Alvarez Ever, Riccio Yauri Luis \\ Instituto de Investigación de la Facultad de Ingeniería Química
}

\begin{abstract}
RESUMEN
Las aguas residuales municipales que se generan en todas las ciudades no están siendo tratadas, las cuales generan problemas ambientales. Una materia orgánica fácilmente biodegradable es un carbohidrato como la lactosa que nos proporciona una metanización sencilla y de fácil modelamiento matemático e interpretación numérica.Los resultados obtenidos fueron que el modelo que se propone consta de 5 ecuaciones diferencias ordinarias y 4 ecuaciones algebraicas. La eficiencia de remoción que se obtuvo fue $95 \%$ para una carga volumétrica de $4 \mathrm{~g}$ materia orgánica/l. La formación de metano y dióxido de carbono ha sido muy baja por la inhibición del proceso de metanogénesis debido a una alta concentración de hidrógeno en el reactor.
\end{abstract}

Palabra clave: Reactor Anaerobio de Flujo Ascendente, Metanogénesis.

\begin{abstract}
Municipal wastewater generated in all cities is not being treated, which cause environmental problems. A readily biodegradable organic matter is a carbohydrate such as lactose which provides a simple and easy mathematical modeling and numerical interpretation. The results were that the proposed model consists of 5 different ordinary equations and 4 algebraic equations. The removal efficiency obtained was $95 \%$ for a volumetric charge of organic material $4 \mathrm{~g} / \mathrm{l}$. The formation of methane and carbon dioxide has been very low by the inhibition of methanogenesis process due to a high concentration of hydrogen in the reactor
\end{abstract}

Key words: Upflow Anaerobic Sludge Blanket, methanogenesis.

\section{INTRODUCCIÓN}

La búsqueda de tecnologías apropiadas para el tratamiento de las aguas residuales municipales de grandes o medianas ciudades constituye un reto que deben afrontar los países subdesarrollados (Collazos C., 1993), especialmente en ciudades altoandinas de alta precipitación, donde el sistema de recolección de las aguas residuales municipales están mezclados, entre aguas residuales domésticas que contienen proteínas, carbohidratos, grasas y aceites, sólidos suspendidos, nitrógeno, fósforo, microorganismos patógenos y fecales, surfactantes, azufre y sulfuro de hidrógeno; con aguas pluviales que tienen plomo, zinc, cadmio y residuos de combustibles y con las aguas industriales conteniendo sólidos suspendidos, materia orgánica biodegradable en alta concentración, nitrógeno, fósforo, metales pesados, sustancias tóxicas, sustancias recalcitrantes, azufre y $\mathrm{pH}$ extremos. Por las consideraciones anteriores, las aguas residuales municipales se componen básicamente de un $99.9 \%$ de agua en su estado conocido como agua potable y de un $0.1 \%$ constituidos de sólidos disueltos y suspendidos (Ruiz I, 1996). Como resultado de la combinación de diferentes tipos de aguas residuales, estas se vuelven complejas que dificultan la implementación de un sistema de tratamiento de las aguas residuales municipales.

\section{MATERIAL Y MÉTODOS}

Material
El modelo matemático consiste de 5 ecuaciones 
diferenciales ordinarias y 4 ecuaciones algebraicas, como se muestra en el anexo del presente informe. En el balance de biomasa se ha considerado que estos son intermitentes, de tal manera que sea una acumulación transiente de biomasa y una purga parcial periódica. También en el modelamiento se está considerado el efecto del pH sobre la velocidad específica máxima de crecimiento. Del mismo modo, en el modelo se considera el comportamiento del fluido tipo mezcla completa.

La resolución del modelo matemático integrado por las ecuaciones diferenciales y algebraicas se realizó mediante el programa del Software Matlab versión 6.12. La integración de las ecuaciones diferenciales se realiza por el método de RungeKutta (Carnahan et al., 1969) con 4 pasos de integración. Se utiliza una computadora personal Pentium Corel i7 por lo que cada simulación tarda poco tiempo de procesamiento.

\section{Equipos}

Computadora INTEL® COREL 2 DUO 2.44 GHz

\section{MÉTODO}

La biodegración de materia orgánica en un reactor anaerobio es bastante compleja y su representación matemática es difícil de resolver mediante una simulación computarizada para explicar los fenómenos biológicos. Para simplificar estos análisis de interpretación de los resultados de la simulación numérica, estamos proponiendo una biodegración de la lactosa en un reactor anaerobio RAFA. Según Chartrain y Zeikus (1986) el $85 \%$ de la lactosa se transforma en ácido láctico y éste, sin acumularse, se convierte en ácido acético. Sus conclusiones están resumidas en la figura 2.1. Estas reacciones ocurren a valores de $\mathrm{pH}$ de 7 y para una $\mathrm{Bv} 2 \mathrm{~g} / \mathrm{l}$. Es interesante ver la coincidencia de Chartrain y Zeikus con Kisaalita et al. (1989) pues este último observó que a bajas velocidades de carga orgánica $\left(B v=12 \mathrm{~g} / /^{*} \mathrm{~d}\right)$ y $\mathrm{pH}=6$ se produce metano y no se detecta ácido láctico, mientras que al aumentar la $\mathrm{Bv}$ o bajar el $\mathrm{pH}$, el metano disminuye hasta desaparecer y aparece lactato. Así, mientras no hay bacterias metanogénicas el metabolismo se dirige $50 \%$ al butirato y resto hacia acetato y propionato.

\section{RESULTADOS}

Se considera una interrelación entre las tres fases reactivas, la sólida o de microorganismos, la gaseosa y la líquida, en donde todas contribuyen para llevar a cabo las reacciones y el equilibrio fisicoquímico. En los balances de nasa se ha considerado que en el reactor el fluido tiene un comportamiento de flujo como si fuera completamente mezclado.

A. Cálculo de la velocidad específica de crecimiento $(\mu)$

a) Para la bacteria acidogénica $(\mu 1)$

$$
\mu_{1}=\mu_{\max 1} \frac{S}{K_{S}+S}-b_{1}
$$

b) Para la bacteria metanogénica $(\mu 2)$

$$
\mu_{2}=\mu_{m a 2} \frac{R(p H)}{1+\frac{K_{S_{2}}}{a^{*} c}+\frac{a^{*} c}{K_{i a}}}-b_{2}
$$

Dónde:

$$
R(p H)=-p H^{2}+14 * p H-48
$$

Y la fracción no disociada es:

$$
C=\frac{1}{1+10^{p H-p K a}}
$$

\section{B. Balance de generación de biomasa}

a) Para la bacteria acidogénica ( $x 1)$

$$
\frac{d x_{1}}{d t}=\mu_{1} * x_{1}-P_{1}
$$

b) Para la bacteria metanogénica (x2)

$$
\frac{d x_{2}}{d t}=\mu_{2} * x_{2}-P_{2}
$$




\section{Balance de consumo de sustrato}

a) Para consumo de lactosa (s)

$$
\frac{d s}{d t}=D\left(s_{0}-s\right)-\frac{\mu_{1}}{y_{1}} * x_{1}
$$

b) Para consumo de AGV (a)

$$
\frac{d a}{d t}=D\left(a_{0}-a\right)+\frac{\mu_{1}}{y_{3}} * x_{1}-\frac{\mu_{2}}{y_{2}} * x_{2}
$$

D. Balance del equilibrio de bicarbonato (equilibrio físico-químico)

a) Para el bicarbonato (bc)

$$
\frac{d b_{c}}{d t}=D\left[b_{C_{0}}-b_{C}-\left(\frac{a}{\rho(1-C)}\right)\right]-\frac{\mu_{1}}{y_{3}} * \frac{x_{1}}{P M}
$$

b) Para el pH

$$
p H=p K a+\log \left(\frac{b_{c}}{\mathrm{CO}_{2}}\right)
$$

c) Para la concentración de $\mathrm{CO} 2$

$$
\mathrm{CO}_{2}=\mathrm{pCO}_{2} * \mathrm{H}_{e}
$$

\section{E. Velocidades de flujo de biogas}

a) Para el hidrógeno

$$
Q_{H_{2}}=\frac{\mu_{1}}{y_{7}} * x_{1}
$$

b) Para el metano

$$
Q_{C_{4}}=\frac{\mu_{2}}{y_{4}} * x_{2}+Q_{H_{2}} * k_{8}
$$

c) Para el dióxido de carbono

$$
Q_{\mathrm{CO}_{2}}=\frac{\mu_{1}}{y_{5}} * x_{1}+\frac{\mu_{2}}{y_{6}} * x_{2}-Q_{H_{2}} * k_{9}
$$

\section{DISCUSIÓN}

Las cinco ecuaciones diferenciales ordinarias simultáneas se resolvieron utilizando el método numérico Runge-Kutta de cuarto orden, y conjuntamente con las ecuaciones simultáneas no lineales se realizo la calibración del modelo de tal forma que reprodujiese valores cercanos a la realidad, todo el proceso se realiza por iteración continua en una computadora. Algunos coeficientes estequiométricos de las reacciones biológicas fueron determinadas a partir de la formulación de las relaciones estequiométricas y otros fueron hallados de la literatura; los demás parámetros y coeficientes para la simulación fueron encontrados en la bibliografía. En la calibración del modelo se utilizaron los parámetros que se muestran en la tabla 2.3. Todos los resultados de la simulación en computadora se presentan en forma de gráficos.

\begin{tabular}{|c|c|c|c|}
\hline Parámetro & Unidades & Valor & Referencias \\
\hline$\mu_{\max 1}$ & $\mathrm{~d}^{-1}$ & 25,2 & Mosey F. \\
\hline $\begin{array}{l}\mu_{\max 1} \\
\mu_{\max 2}\end{array}$ & $d^{-1}$ & 0,45 & Lema J. \\
\hline $\begin{array}{c}\mu_{\max 2} \\
\mathrm{~K}_{\mathrm{s} 1}\end{array}$ & g DQO// & 2,924 & Monroy O. \\
\hline $\mathrm{K}_{\mathrm{s} 2}$ & $\mathrm{~g} D Q O / /$ & 0,003 & Fdz-Polanco \\
\hline $\mathrm{K}_{\mathrm{ia}}^{\mathrm{s} 2}$ & g DQO/I & 4,8 & Pavlostathis \\
\hline$y_{1}$ & $\mathrm{~g}_{1} / \mathrm{g}$ DQO & 0,15 & y G. \\
\hline$y_{2}$ & $\mathrm{~g} \mathrm{x}_{2} / \mathrm{g} \mathrm{AGV}$ & 0,041 & Monroy O. \\
\hline$y_{3}$ & $g x_{1} / g$ AGV & 0,7513 & Lawrence y \\
\hline$b_{1}$ & $d^{-1}$ & 1,09 & McCarty \\
\hline$b_{2}$ & $\mathrm{~d}^{-1}$ & 0,0025 & Monroy O. \\
\hline$y_{4}$ & $\mathrm{~g} \mathrm{x}_{2} / / \mathrm{CH}_{4}$ & 0,0255 & Mosey F. \\
\hline$y_{5}$ & $\mathrm{~g} \mathrm{x}_{1} / / \mathrm{CO}_{2}$ & 1,1695 & Monroy O. \\
\hline$y_{6}$ & $\mathrm{~g} \mathrm{x}_{2} / \mathrm{CO}_{2}$ & 0,0255 & Monroy 0. \\
\hline$y_{7}$ & $\mathrm{~g} \mathrm{x}_{1} / / \mathrm{H}_{2}$ & 0,7653 & Monroy O. \\
\hline $\mathrm{k}_{8}$ & $\mathrm{I} \mathrm{CH}_{4} / \mathrm{l} \mathrm{H}$ & 0,25 & Monroy $\mathrm{O}$. \\
\hline $\mathrm{k}_{9}$ & I $\mathrm{CO}_{2} / \mathrm{l} \mathrm{H}$ & 0,25 & Monroy O. \\
\hline $\mathrm{P}_{\mathrm{CO} 2}$ & atm & 0,3 & Monroy O. \\
\hline $\mathrm{He}$ & mol & 0,0246 & Monroy O. \\
\hline & $\mathrm{CO}_{2} / \mathrm{atm}^{*} \mathrm{I}$ & & Cuervo- \\
\hline & & & Lopez \\
\hline & & & López \\
\hline
\end{tabular}
El modelo que se propone es adecuado para predecir la biodegradación de lactosa y la eficiencia de eliminación de la DQO como se aprecian en el gráfico 1. El rápido consumo de la lactosa se confirma por el crecimiento tan rápido de las bacterias acidogénicas (gráfico 2).

Tabla 2.3. Parámetros utilizados en la simulación 
Gráfico 1. Eficiencia de remoción de lactosa

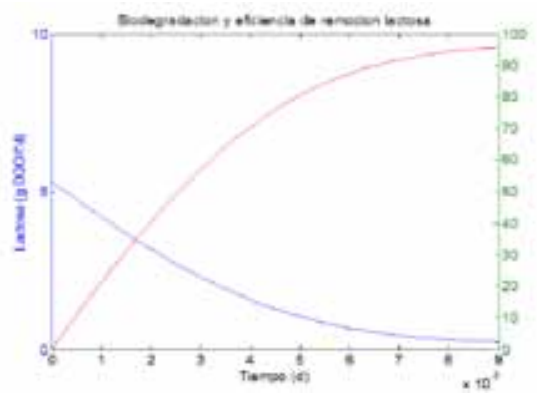

Esto nos indica que la primera etapa de acidogénesis se realiza en forma inmediata ya que tan solo se necesita de 13 minutos para biodegradar la lactosa. Sin embargo, el modelo falla al predecir el balance fisicoquímico en el reactor, pues como se aprecia en el gráfico 3 , la curva de predicción del pH disminuye debido al aumento de la concentración de $\mathrm{H} 2$ en el medio, como resultado de la degradación de la lactosa para formar ácidos grasos volátiles y liberan $\mathrm{H} 2$.

El pH disminuye desde un valor inicial de 7, hasta llegar a un $\mathrm{pH}$ de 6 , en donde la lactosa llega a tener una concentración de $1,8 \mathrm{~g} \mathrm{DQO} / /^{*} \mathrm{~d}$ y es aquí donde comienza a desestabilizarse el reactor porque ya no hay formación de bacterias metanogénicas por inhibición de la concentración de H2. En el modelo se ha tratado de controlar el $\mathrm{pH}$ dentro del rango de $6-8$, pero no fue así, es por ello que se ha tenido que ajustar nuevamente a un $\mathrm{pH}$ 7. En este tipo de reactores es importante añadir bicarbonato para mantener un nivel de $1,5 \mathrm{meq} / /^{*} \mathrm{~d}$ en promedio para mantener el equilibrio. Los resultados nos indican que hay una alta producción de AGV alrededor de $22 \mathrm{meq} / /^{*} \mathrm{~d}$.

Gráfico 2. Crecimiento de las bacterias acidogénicas

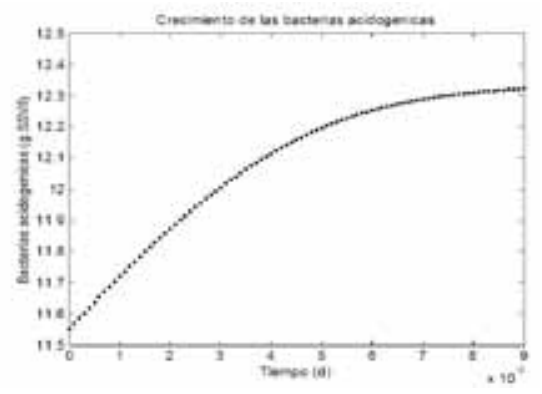

Gráfico 3. Concentración de los ácidos grasos volátiles

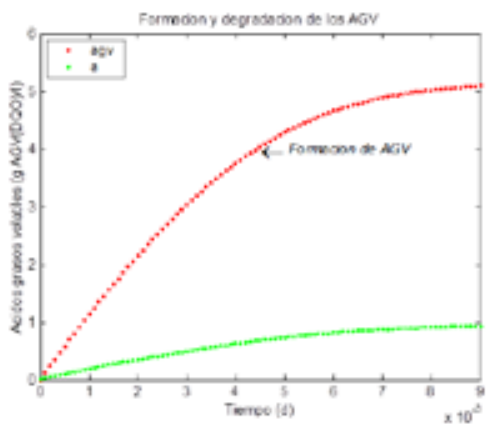

\section{CONCLUSIONES}

1. El tiempo de residencia hidráulica en el reactor es de $13 \mathrm{~h}$, lo que implica que el volumen de diseño no tendrá problemas para trabajar con otras cargas orgánicas.

2. El modelo matemático para la degradación de lactosa está integrado de 5 ecuaciones diferenciales ordinarias y 4 ecuaciones algebraicas, esta combinación de ecuaciones han sido solucionados mediante técnicas del cálculo numérico y han sido programados en el software Matlab.

3. El modelo matemático predice muy bien la degradación de lactosa hasta ácidos grasos volátiles, proporcionándonos una descripción clara de los fenómenos y procesos que ocurren en la digestión anaerobia.

4. Según el modelo matemático la producción de metano y dióxido de carbono es muy baja debido a la inhibición producida por el descenso del $\mathrm{pH}$ a valores por debajo de 7; es decir, por que existe una sobre carga de ácidos grasos volátiles y formación de $\mathrm{H} 2$.

\section{REFERENCIAS BIBLIOGRÁFICAS}

1. Abdel-Halim W., El-Sayed W., Halim H., and Rosenwinkel K., "Municipal Wastewater Treatment in Developing Countries Comparable Alternative Anaerobic Cost-Effective Systems", Housing and Building National Research Center 
(HBNRC), Egipto, 2009.

2. Aiyuk, S., Odonkor, P., Theko, N., Haandel, A. and Verstraete, W., "Technical problems ensuing from UASB reactor application in domestic wastewater treatment without pre-treatment", International journal of environmental science and development, 2010.

3. Aldana, G. and Pérez, J., "Mean hydraulic retention time and pathways flow simulation trough out a physical model for a WSPs and an UASB", Process Biosyts Eng., 2010.

4. Cheol, G. and Noike, T., "Effect of rapid temperature change and HRT on anaerobic acidogenesis", Departament of Civil Engineering, Tohoku University, Japan, 1997.

5. Elías, A., Barona, A., Ormazabal, J., Ibarra, G. and Camano, J., "Anaerobic treatment of acidifield and non-acidified substrata in UASB reactors", Journal of Chemical Technology and Biotechnology, España, 1999.

6. Elmitwalli T., Shalabi M., Wendland C. and Otterpohl R., "Grey water treatment in UASB reactor at ambient temperature", Department of Civil Engineering, Benha High Institute of Technology, Egipto, 2007.

7. Ingaruca E., "Diagnóstico del tratamiento de las aguas residuales en la ciudad de Huancayo", CIP, Huancayo. 2004.

8. Ingaruca E., "Especialista en Tratamiento y Reuso de Agua Residual para el Proyecto Fortalecimiento de la Gestión Ambiental en el Gobierno Regional y Municipio de la Región Junín", Gobierno Regional de Junín, Huancayo 2010.

9. Kripa S. and Viraraghavan T., "Impact of temperature on performance, microbiological, and hydrodynamic aspects of UASB reactors treating municipal wastewater", Department of Civil Engineering, University of New Brunswick, Canada, 2008.

10. Lema, J.M., "Operación y Control de Digestores
Anaerobios", Dpto. de Ingeniería Química, Universidad de Santiago de Compostela, España,1992.

11. Mahmound, N., "High Strength sewage treatment in a UASB reactor and an integrated UASB-digester system", Institute of Environmental and Water studies (IEWS), Birzeit University, Palestine, 2008.

12. Monroy H. O., Gómez H. J. "Curso: Procesos Biológicos para el Tratamiento de Aguas Residuales", Universidad de Concepción-Chile, Departamento de Ingeniería Química, Octubre, 1995.

13. Monroy O., Vázquez F., "Anaerobic-aerobic treatment of cheese wastewater with nacional technology in México: The case of El Sauz", Water Science Technology, 32,12:149-156, 1995.

14. Noyola A., Anaerobic Digestion in Latin America: "Anaerobic Digestion Applied to Municipal Wastewater Treatment", Institute of Engineering UNAM-Mexico, 2009.

15. Ruiz, I., Alvarez, J.A., Zapico, C.M., Gómez, M., Presas, J. and Soto, M., "Tratamiento anaerobio de aguas residuales urbanas a temperatura ambiente en digestores UASB de laboratorio y planta piloto", Universidad de Coruña, Departamento de Química Física, España,1996.

16. Solís C., Rodríguez J., Noyola A., "Evaluación del Comportamiento del Color, pH y Alcalinidad en una Planta de Tratamiento Anaerobio", Universidad Autónoma de Estado de México, México, 2005.

17. Tessele F., Monteggia L.O. and Rubio J., "Treatment of municipal wastewater UASB reactor effluent by unconventional flotation and UV disinfection", Instituto de Pesquisas Hidra' ulicas. Universidade Federal do Rio Grande do Sul, Brasil, 2005. 